\title{
Single Crystal Organic Field-effect Transistors Based on 2,8-Diphenyl and Dinaphthyl Chrysenes
}

\author{
Yoshihito Kunugi ${ }^{1 *}$, Tatsuya Arai ${ }^{1}$, Norihito Kobayashi ${ }^{1}$, Hiroyuki Otsuki ${ }^{2}$, Toru \\ Nishinaga $^{3}$ and Kazuo Okamoto ${ }^{2}$ \\ I Department of Applied Chemistry, Faculty of Engineering, Tokai University, \\ 4-1-1 Kitakaname, Hiratsuka 259-1292, Japan \\ *ykunugi@keyaki.cc.u-tokai.ac.jp \\ ${ }^{2}$ Reseach Laboratory, Ushio Chemix Corporation, \\ 2252-1 Goudo, Omaezaki 437-1613, Japan \\ ${ }^{3}$ Department of Chemistry, Graduated School of Science and Engineering, Tokyo \\ Metropolitan University, 1-1 Minamiosawa, Hachioji, Tokyo 192-0397, Japan
}

\section{Keywords: Organic Single Crystal, Organic Field-effect Transistor, Chrysene derivatives, Highly-mobility Organic Transistor}

\section{Introduction}

Organic semiconductors have attracted significant attention in materials science because of their potential application in large-area, low-cost and flexible electronic devices such as optical displays, chemical and physical sensors and radio frequency identification tags [1-5]. In particular, organic field-effect transistors (OFETs), the fundamental building blocks of organic-based circuits, are one of the most important topics. Low-cost production via a printing process and large area coverage are among the attractive research topics. However, disorder and grain boundaries in organic thin films are intrinsic properties of organic semiconductors and degrade device performance. Therefore, to study the
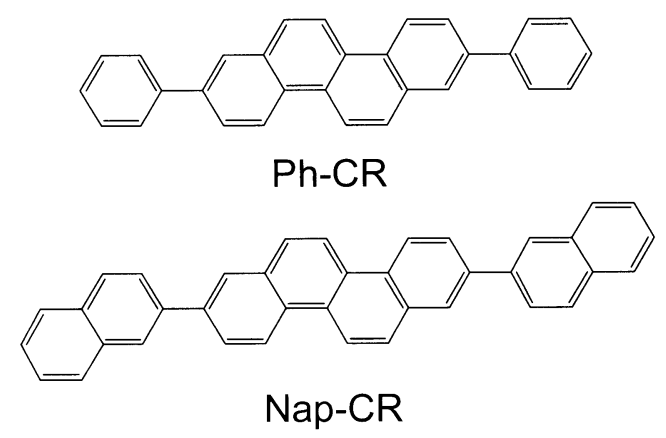

Figure 1. Molecular structures of chrysene derivatives. intrinsic charge transport properties of organic semiconductors and conduct correlation studies of crystal structure and FET performance, single crystal devices are needed. Single crystal OFETs perform better than thin film transistors, because of high molecular ordering and the absence of boundaries in the crystal. Great effort has been devoted to the development of newly fused $\pi$-conjugated organic semiconductors for high-performance organic single crystal transistors [6-11].

We recently synthesized a series of 2,8-disubstituted chrysene derivatives and applied them to organic thin film transistors [12]. Chrysene derivatives based thin film transistors showed typical $p$-channel FET characteristics, and their performance in devices was strongly affected by the molecular ordering and grain size of the organic thin films. The best field-effect mobility of $4.1 \times 10^{-2} \mathrm{~cm}^{2} \mathrm{~V}^{-1} \mathrm{~s}^{-1}$ with a current on/off ratio of $10^{5}$ was obtained for 2,8-diphenyl chrysene (Ph-CR) based transistors. In this study, we fabricated and measured chrysene based single crystal OFETs to examine the intrinsic properties of chrysene derivatives.

\section{Experimental}

$\mathrm{Ph}$-CR and 2,8-bis(2-naphthyl)chrysene (Nap-CR) were synthesized by a previously reported procedure $[12,13]$. All compounds were

Received March 29, 2011

Accepted May 13, 2011 


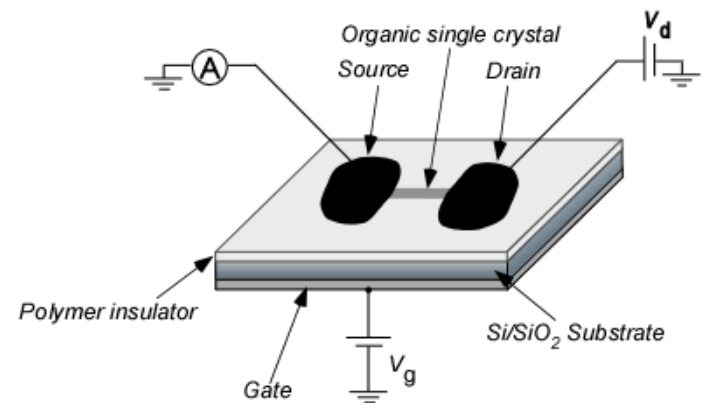

Figure 2. Schematic illustration of the single crystal OFET device.

characterized by a combination of ${ }^{1} \mathrm{H} N \mathrm{NMR}$ (400 $\mathrm{MHz}),{ }^{13} \mathrm{C}$ NMR $(100 \mathrm{MHz}), \quad I R$ and $\mathrm{MS}$ spectroscopy. The resulting chrysene derivatives were purified by vacuum sublimation before use. Other materials were reagent grade or better and were used as received.

Organic single crystals were grown by horizontal physical vapor transport in an argon gas flow [14]. The evaporating material was heated to $280{ }^{\circ} \mathrm{C}$ in the hot zone of a two-zone furnace. The second zone of the furnace was maintained at 230 ${ }^{\circ} \mathrm{C}$. The crystals grew spontaneously on the wall of a glass tube in the colder zone of the furnace.

Top-contact type single crystal OFET devices were prepared by crystal lamination (Figure 2) [9-11]. A s substrates for the lamination, we used an $\mathrm{n}^{+}$-doped Si wafer with $210 \mathrm{~nm}$ thick thermally grown $\mathrm{SiO}_{2}$, which was treated with a thin film (ca. $30 \mathrm{~nm}$ ) of a polymer insulator such as poly (methyl methacrylate) (PMMA) or CYTOP (A sahi Glass Corp.). The polymer insulators were spin-coated at $2000 \mathrm{rpm}$ and then dried at $70{ }^{\circ} \mathrm{C}$ and annealed at $100{ }^{\circ} \mathrm{C}$ for $3 \mathrm{~h}$ (PMMA) or dried at $90{ }^{\circ} \mathrm{C}$ and baked at $200{ }^{\circ} \mathrm{C}$ for $1 \mathrm{~h}$ (CY TOP) on a hot plate in $\mathrm{N}_{2}$ atmosphere $[15,16]$. Shiny, thin and detected-free chrysene single crystals were carefully selected and laminated on top of the substrates. Source and drain electrodes were applied with a colloidal graphite paste.

The electrical properties of the OFET devices were measured by using an Agilent $4155 \mathrm{C}$ semiconductor parameter analyzer under vacuum at room temperature. The field-effect mobility $\left(\mu_{\mathrm{FET}}\right)$ was extracted from the saturated regime of the drain current $\left(I_{d}\right)$ using the equation $I_{d}=$ $\left(W C_{i} / 2 L\right) \mu_{F E T}\left(V_{g}-V_{t h}\right)^{2}$, where $C_{i}$ is capacitance of the insulator, $V_{d}$ and $V_{\text {th }}$ are the gate and threshold voltages, respectively. The current on/off ratio $\left(I_{\text {on }} / I_{\text {off }}\right)$ was determined from the maximum $\left(I_{\text {on }}\right)$ and minimum $\left(I_{\text {off }}\right)$ values of the $I_{d}$. (a)

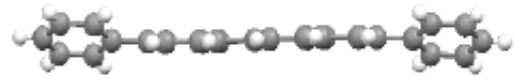

(b)

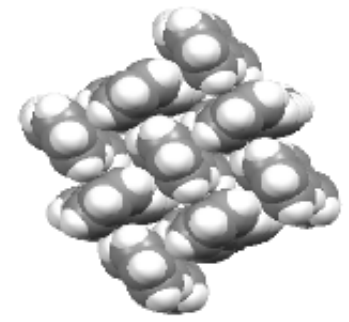

Figure 3. (a) Molecular and (b) packing structures of $\mathrm{Ph}-\mathrm{CR}$.

The X-ray crystal structure analysis was performed on a Rigaku RAXIS RAPID imaging plate area detector with graphite monochromated Mo-K $\alpha(\lambda=0.71069 \AA)$ radiation at $-180{ }^{\circ} \mathrm{C}$. Molecular orbital (MO) calculations were carried out by Density Functional Theory (DFT) methods at the B 3LY P/6-31G(d) level using the Gaussian03 program [17].

\section{R esults and discussion}

Very thin and planar crystals of $\mathrm{Ph}-\mathrm{CR}$ and $\mathrm{N}$ ap-CR were obtained by horizontal physical vapor transportation. To investigate the molecular conformation and stacking of the molecules in the crystal state, $\mathrm{X}$-ray single-crystal analysis was performed. Figure 3 shows molecular and packing structures for single crystal of Ph-CR. The phenyl substituents of $\mathrm{Ph}-\mathrm{CR}$ are tilted $26.4^{\circ}$ from the chrysene plane, and the crystal structure of Ph-CR exhibits a herringbone pattern that is favorable for high performance organic transistors [18, 19]. The packing structure of $\mathrm{Nap}-\mathrm{CR}$ is not solved completely here because of poor crystal thickness.

Figure 4 shows the typical drain current as a function of the applied drain voltage at various gate voltages for OFETs based on Ph-CR and $\mathrm{N}$ ap-CR single crystals whose $\mathrm{SiO}_{2}$ insulator was treated with CYTOP. The devices show a typical p-channel output profile of a metal-oxide-semiconductor FET. The field-effect mobility and the threshold voltage were calculated from the slope and intercept, respectively, of the linear portion of the $\left(-I_{d}\right)^{1 / 2}-V_{g}$ plot. The field-effect characteristics of chrysene based single crystal OFETs with various polymer insulator treatments are summarized in Table 1 . The field-effect characteristics were strongly affected by the applied polymer insulator. The reported 

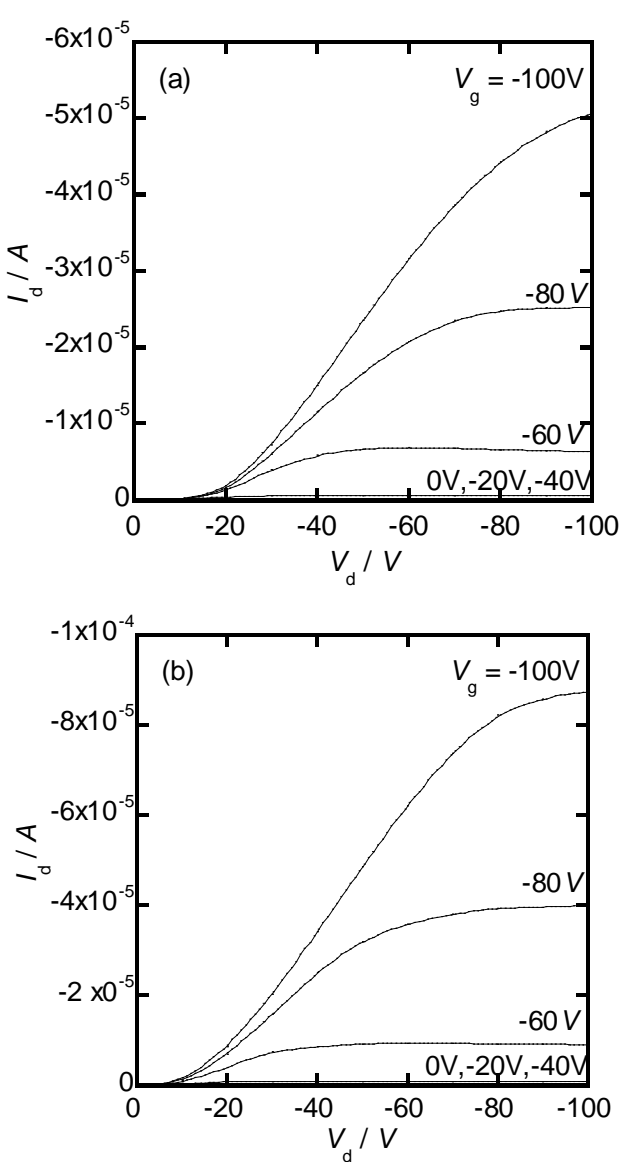

Figure 4. $I_{d}$ Vs. $V_{d}$ characteristics at various gate biases for OFET devices based on (a) Ph-CR and (b) $\mathrm{Nap}-\mathrm{CR}$ single crystals whose $\mathrm{SiO}_{2}$ insulator was treated with CY TOP.

contact angles with water for $\mathrm{SiO}_{2}, \mathrm{PMMA}$ and CY TOP are $20^{\circ}, 68^{\circ}$ and $108^{\circ}$, respectively [20, 21]. Hydrophobic insulators are known to be favorable for high performance thin-film and single crystal OFETs [8, 21, 22]. The best FET characteristics of $\mu_{\mathrm{FET}}=2.2 \mathrm{~cm}^{2} \mathrm{~V}^{-1} \mathrm{~s}^{-1}$ and $\mathrm{I}_{\text {on }} / /_{\text {off }}=$ $10^{6}$ were obtained in the CY TOP treated device for the Nap-CR single crystal.

To obtain further information on the relationship between molecular structure and FET characteristics, we examined the energy levels of

Table 1. Field-effect characteristics of chrysene based single crystal OFETs with various polymer insulator treatments.

\begin{tabular}{ccccc}
\hline Compound & $\begin{array}{c}\text { Polymer } \\
\text { insulator }\end{array}$ & $\begin{array}{c}\mu_{\text {FET }} / \\
\mathrm{cm}^{2} \mathrm{~V}^{-1} \mathrm{~S}^{-1}\end{array}$ & $\mathrm{I}_{\text {on }} / \mathrm{I}_{\text {off }}$ & $\begin{array}{c}\mathrm{V}_{\text {th }} \\
/ \mathrm{V}\end{array}$ \\
\hline Ph-CR & - & 0.29 & $10^{4}$ & -20 \\
Ph-CR & PM M A & 0.37 & $10^{4}$ & -22 \\
Ph-CR & CY TOP & 1.6 & $10^{3}$ & -35 \\
Nap-CR & - & 0.54 & $10^{3}$ & -18 \\
Nap-CR & PM M A & 0.96 & $10^{5}$ & -17 \\
Nap-CR & CY TOP & 2.2 & $10^{6}$ & -33 \\
\hline
\end{tabular}

(a)

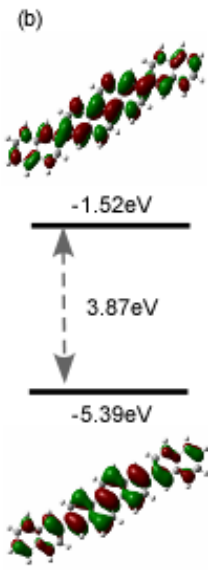

Figure 5. Calculated energy diagram and frontier orbitals of (a) Ph-CR [12] and (b) Nap-CR.

the chrysene derivatives by MO calculations. The electronic properties of unsubstituted chrysene, $\mathrm{Ph}-\mathrm{CR}$ and $\mathrm{N}$ ap-CR were calculated with the DFT MO calculation [B $3 L Y P / 6-31 G(d)$ ] embedded in the Gaussian 03 software package. The resulting molecular orbitals and energy diagrams are shown in Figure 5 [12]. The HOMO energy levels of $\mathrm{Ph}-\mathrm{CR}$ and Nap-CR are slightly higher than that of unsubstituted chrysene (-5.50 eV), and the LUM 0 energy levels of Ph-CR and Nap-CR are slightly lower than that of the unsubstituted chrysene (-1.25 $\mathrm{eV})$. The band gap energy for the Nap-CR (3.87 $\mathrm{eV})$ are slightly narrower than that for Ph-CR (4.03 $\mathrm{eV})$, which is favorable for charge transport between molecules.

\section{Conclusion}

W e successfully fabricated single crystal OFETs based on chrysene derivatives Ph-CR and Nap-CR. The single crystal chrysene based OFETs showed typical p-channel FET characteristics, and the highest field-effect mobility of $2.2 \mathrm{~cm}^{2} \mathrm{~V}^{-1} \mathrm{~s}^{-1}$ with an on/off current ratio of $10^{6}$ was obtained for Nap-CR.

\section{Acknowledgement}

This study was financially supported in part by a Grant-in-Aid for Science Research from the M inistry of Education, Culture, Sports, Science and Technology of Japan.

\section{R eferences}

1. A. C. A rias, J. D. M ack enzie, I. M aCulloch, J. Rivnay and A. Salleo, Chem. Rev., $\mathbf{1 1 0}$ (2010) 3.

2. G. Gelinck, P. Heremans, K. Nomoto and T. D. 
Anthopoulos, Adv. Mater, 22 (2010) 3778.

3. T. Someya, A. Dodabalapur, J. Huang, K. C. See and H. E. Katz, Adv. Mater., 22 (2010) 3799.

4. A. Caboni, E. Orgiu, M. Barbaro and A. Bonfiglio, IEEE Sens. J., 9 (2009) 1963.

5. P. Andersson, R. Forchheimer, P. Tehrani and M. Berggren, Adv. Func. Mater., 17 (2007) 3074.

6. V. C. Sundar, J. Zaumseil, V. Podzorov, E. Menard, R. L. Willett, T. Someya, M. E. Gershenson and J. A. Rogers, Science, 303 (2004) 1644.

7. J. Takeya, M. Yamagishi, Y. Tominari, R. Hirahara, Y. Nakazawa, T. Nishikawa, T. Kawase, T. Shimoda and S. Ogawa, Appl. Phys. Lett., 90 (2007) 102120.

8. S. Haas, Y. Takahashi, K. Takimiya and T. Hasagawa, Appl. Phys. Lett., 95 (2009) 022111.

9. Y. Kunugi, T. Arai, M. Tsutsui, H. Maeda and K. Okamoto, J. Photopolym. Sci. Technol., 23 (2010) 323.

10. R. Zeis, C. Kloc, K. Takimiya, Y. Kunugi, Y. Konda, N. Niihara and T. Otsubo, Jap. J. Appl. Phys., 44 (2005) 3712.

11. K. Yamada, T. Okamoto, K. Kudoh, A. Wakamiya, S. Yamaguchi and J. Takeya, Appl. Phys. Lett., 90 (2007) 072102.

12. Y. Kunugi, M. Ikari and K. Okamoto, ECS Trans., 25 (2009) 11.

13. B. K. Banik, M. S. Venkatraman, I. Banik and K. Basu, Tetrahedron. Lett., 45 (2004) 4737.

14. R. A. Laudise, Ch. Kloc, P. G. Simpkins and T. Siegrist, J. Cryst. Growth., 187 (1998) 449.

15. T. Takenobu, K. Watanabe, Y. Yomogida, H. Shimotani and Y. Iwasa, Appl. Phys. Lett., 93 (2008) 073301.

16. M. Uno, Y. Tominari and J. Takeya, Org.
Electron., 9 (2008) 753.

17. M. J. Frisch, G. W. Trucks, H. B. Schlegel, G. E. Scuseria, M. A. Robb, J. R. Cheeseman, J. A. Montgomery Jr., T. Vreven, K. N. Kudin, J. C. Burant, J. M. Millam, S. S. Iyengar, J. Tomasi, V. Barone, B. Mennucci, M. Cossi, G. Scalmani, N. Rega, G. A. Petersson, H. Nakatsuji, M. Hada, M. Ehara, K. Toyota, R. Fukuda, J. Hasegawa, M. Ishida, T. Nakajima, Y. Honda, O. Kitao, H. Nakai, M. Klene, X. Li, J. E. Knox, H. P. Hratchian, J. B. Cross, V. Bakken, C. Adamo, J. Jaramillo, R. Gomperts, R. E. Stratmann, O. Yazyev, A. J. Austin, R. Cammi, C. Pomelli, J. W. Ochterski, P. Y. Ayala, K. Morokuma, G. A. Voth, P. Salvador, J. J. Dannenberg, V. G. Zakrzewski, S. Dapprich, A. D. Daniels, M. C. Strain, O. Farkas, D. K. Malick, A. D. Rabuck, K. Raghavachari, J. B. Foresman, J. V. Ortiz, Q. Cui, A. G. Baboul, S. Clifford, J. Cioslowski, B. B. Stefanov, G. Liu, A. Liashenko, P. Piskorz, I. Komaromi, R. L. Martin, D. J. Fox, T. Keith, M. A. Al-Laham, C. Y. Peng, A. Nanayakkara, M. Challacombe, P. M. W. Gill, B. Johnson, W. Chen, M. W. Wong, C. Gonzalez and J. A. Pople, Gaussian 03, Gaussian, Inc., Wallingford CT (2004).

18. T. Yamamoto and K. Takimiya, J. Am. Chem. Sc., 129 (2007) 2224.

19. T. Hasegawa and J. Takeya, Sci. Technol. Adv. Mater., 10 (2009) 024314.

20. X. Liang, S. Liu, H. Liu, X. Liu and S. Jiang, J. Sep. Sci, 33 (2010) 3304.

21. Y. Fujisaki, M. Mamada, D. Kumaki, S. Tokito and Y. Yamashita, Jap. J. Appl. Phys., 48 (2009) 111504.

22. W. H. Lee, J. H. Cho and K. Cho, J. Mater. Chem., 20 (2010) 2549. 